\title{
ESTABLISHING SPV FOR POWER PROJECTS IN ASIA: AN ANALYSIS OF CRITICAL FINANCIAL AND LEGAL FACTORS
}

\author{
Abu Naser Chowdhury ${ }^{1}$, Po-Han Chen², Robert Lee Kong Tiong ${ }^{3}$ \\ ${ }^{1}$ Nanyang Technological University (NTU), Singapore \\ ${ }^{2}$ Department of Civil Engineering, National Taiwan University (NTU), Taiwan \\ ${ }^{3}$ School of Civil and Environmental Engineering, \\ Nanyang Technological University (NTU), Singapore \\ E-mails:1abun0001@e.ntu.edu (correspondingauthor); \\ 2pohanchen@ntu.edu.tw; ${ }^{3}$ clktiong@ntu.edu.sg \\ Received 05 February 2011; accepted 15 November 2011
}

\begin{abstract}
One of the main reasons for the success of Public Private Partnership (PPP) projects is the creation of separate commercial venture named 'Special Purpose Vehicle' (SPV). SPV provides a good framework for raising funds, linking participants legally and assuring supply, production and marketing of products. SPV brings together various parties like lenders, financial institutions, public sector and export credit agencies, guarantors, suppliers and off-takers. There is often a lack of precedents to identify factors of SPV and the process is further hampered by undeveloped financial and legal structures of a country. A number of factors need to be considered before setting up of SPV for PPP projects. A questionnaire survey was thus conducted to explore the most important factors in setting up SPV for PPP power projects from a wide range of personnel involved in the PPP processes in Asia. The objective of this paper is to investigate the current practice of setting up SPV and associated legal and financial impacts onto it. Analysis of the response data reflects experts' opinion in identifying the influential factors and the choices in setting up SPV for PPP power projects in Asia. The findings of the paper would help the public sector in implementing PPP power policy development and private sector sponsors in managing the projects.
\end{abstract}

Keywords: Special Purpose Vehicle, Public-Private Partnership, power, Asia.

Reference to this paper should be made as follows: Chowdhury, A. N.; Chen, P.-H.; Tiong, R. L. K. 2012. Establishing SPV for power projects in Asia: an analysis of critical financial and legal factors, Journal of Business Economics and Management 13(3): 546-566.

JEL Classification: N75.

\section{Introduction}

More often than not, Public-Private Partnership (PPP) is constructed through a 'Special Purpose Vehicle' (SPV), which acts as a managing and operating company for project(s) as well as the legal body that guarantees concessions from the public authority. A concession agreement is the agreement between government and the SPV for development, construction and operation of specific projects. As part of the conces- 
sion, a SPV owns and operates the facility and collects revenue which is used to repay the financial and investment costs, to maintain and operate the facility, and to make marginal profits (Merna, Smith 1996). Since financing is arranged through the SPV, it is thus said to be the heart of project financing (Tan 2007; IPFA supra note 4). A contractual network revolves around the SPV where each party sets up contracts with the SPV for a specified period of the project (Gatti 2008). All legal and financial agreements with various parties/stakeholders of a project are accorded with the SPV. Thus, it acts as an entity for legal manifestation of a project consortium. The SPV is embraced by lenders, financial institutions, public authorities, export credit agencies, guarantors, suppliers and off-takers where equity comes from a prime contractor, service provider and public authority. Apart from initial share capital subscription, extra funds are raised either through subordinated debt from project participants or senior secured debt from capital markets or from banks. Because of limited liability of equity holders, creation of the SPV allows off-balance sheet financing which means that the debt raised by the promoters (i.e. investors, contractors, subcontractors and suppliers) would not appear in their balance sheet but it would appear only on the balance sheet of the SPV. This situation allows promoting companies to raise extra debt without providing their own assets as collateral (Dias, Ioannou 1995). At the same time, through the SPV, risks of the participating parties can be minimized and the project can also be assessed on its own merits (Bult-Spiering, Dewulf 2006). The liability of the project sponsors is limited to the amount of capital they have injected, plus any obligations individual sponsors may have under the contracts with the SPV. Though the SPV serves different functions for its various participants in a project (as it is bound by many legal and financial agreements), the main objective of the SPV is to obtain funds. Figure 1 shows the SPV and its agreements with various parties.

Another objective of SPV is to minimize the project risks that are assumed by it and to pass them through the contractual structure to stakeholders that are best able to assess and manage risks. The establishment of SPV for capital-intensive projects is prompted by its ability to spread risk and the expanded borrowing capacity for new investment (Devapriya, Alfen 2003). Figure 1 depicts how a SPV is interrelated with various parties in a project. Most of the boxes in the diagram are self-explanatory and the most common agreements surrounding SPV are the loan agreement, off-take or purchase agreement, supply agreement, concession agreement, O\&M agreement, EngineeringProcurement-Construction (EPC) agreement or turnkey agreement and sponsor's support agreement. Participation of Multi-lateral Development Banks (MDBs) and Export Credit Agencies (ECAs) in PPP project has some specific roles. These agencies place strict requirements on the SPV. Due to their ability to potentially mitigate risk, many offshore and domestic banks are willing to participate in a project. It is believed that governments make greater effort to ensure that loans to MDBs are repaid even in difficult times. Similarly, ECAs are also popular stakeholders in the financing of PPP projects. They offer finance, insurance and guarantee repayment of commercial lender financing in case of political risk and/or commercial risk. 


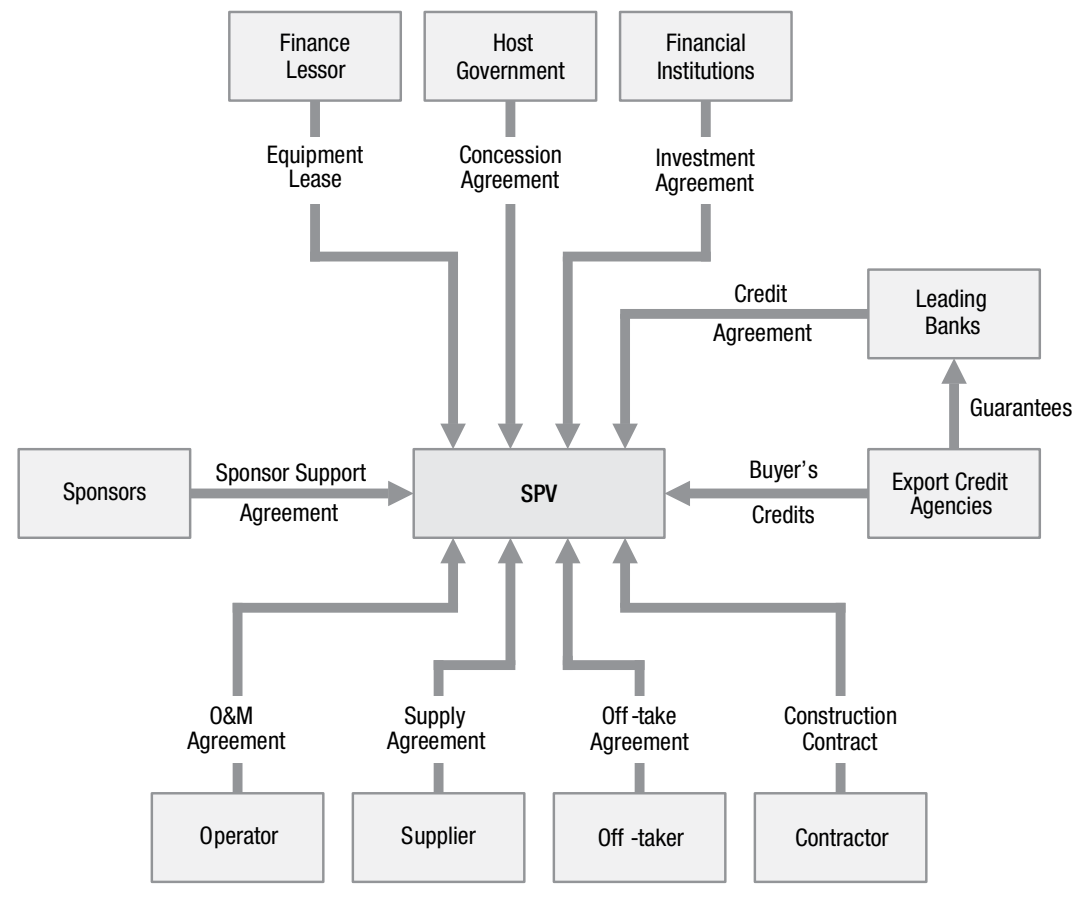

Fig. 1. SPV and its agreements with various parties Source: Sapte (1997)

Structuring of SPV is thus of great importance. Successful structuring of a SPV is therefore essential not only for the success of a project but also for the sanction of loans or debts from banks and/or other financial institutions. Many PPP projects have failed due to the lack of proper identification of potential barriers that the SPV faces, e.g. the legal and financial dimension with long term uncertainties and wide risk sharing portfolios. This paper thus attempts to examine the practice of setting up SPV for PPP power projects in Asia. A questionnaire survey is conducted to explore the most important factors in setting up of SPV for PPP power projects from a wide range of personnel involved in PPP processes in Asia. The objective of this research is therefore to investigate the current practice of setting up SPV of PPP power projects in Asia and the impact of legal and financial issues onto it.

\section{Research methodology}

In order to find out critical financial and legal factors for establishing SPV of PPP power projects in Asia, the study is done in a systematic approach involving three phases: (1) literature review and case investigation of PPP power projects in Asia, (2) questionnaire survey to the experts and experienced practitioners in Asia to identify the most important financial and legal factors, and finally (3) analyze data from completed returned questionnaire with simple statistical techniques like mean, standard deviation, ranking and percentage of responses per factor. Table 1 shows the sources and cases from where the factors for establishing SPV in Asia are collected. 
Table 1. Factors and their sources for establishing SPV in Asia

\begin{tabular}{|c|c|c|}
\hline Factors & Sources & Cases \\
\hline $\begin{array}{l}\text { Contractual } \\
\text { Benchmark } \\
\text { Factors }\end{array}$ & $\begin{array}{l}\text { Khan and Parra (2003), pp. 220-245 } \\
\text { Yescombe (2002), pp. 69-101 } \\
\text { Tiong and Anderson (2003), pp. 235-243 } \\
\text { Kumaraswamy and Zhang (2003), pp. 263-280 } \\
\text { Wang and Tiong (2000), pp. 69-78 } \\
\text { Chowdhury and Chen (2010), pp. 64-88 } \\
\text { Chowdhury and Charoenngam (2009), pp. 267-283 } \\
\text { Hoffman (2008), pp. 17, 209-221 } \\
\text { Finnerty (1996), pp. 60, 65 } \\
\text { Smith et al. (2004), pp. 407-413 } \\
\text { Lang, L. H. P (1998), pp. 241-244 }\end{array}$ & $\begin{array}{l}\text { HubCo, Pakistan } \\
\text { Laibin B, China } \\
\text { Shajiao B, China } \\
\text { Huaibei power plant, } \\
\text { China } \\
\text { AES Lal Pir, Pakistan } \\
\text { Pat Gen, Pakistan }\end{array}$ \\
\hline $\begin{array}{l}\text { Enabling } \\
\text { Environment } \\
\text { Factors }\end{array}$ & $\begin{array}{l}\text { Khan and Parra (2003) } \\
\text { Walsh (2003), pp. 153-180 } \\
\text { Hardcastle and Boothroyd (2003), pp. 31-56 } \\
\text { Davis (2003), pp. } 78-93,94-112 \\
\text { Tiong and Anderson (2003), pp. 225-243 } \\
\text { Hoffman (2008), pp. 23-24, 151 } \\
\text { Qiao et al. (2001) } \\
\text { Zhang et al. (1998) } \\
\text { Jefferies et al. (2002) }\end{array}$ & $\begin{array}{l}\text { Casecnan Water and } \\
\text { Energy Project, the } \\
\text { Philippines } \\
\text { Dabhol Power, India } \\
\text { Paiton 1, Indonesia } \\
\text { Shajiao C, China }\end{array}$ \\
\hline $\begin{array}{l}\text { Guarantee } \\
\text { Factors }\end{array}$ & $\begin{array}{l}\text { Delmon (2009), pp. } 185 \\
\text { ADB: Loans and Political Risk Guarantee (2008) } \\
\text { Davis (2003), pp. } 39-44 \\
\text { Chowdhury and Charoennagm (2009), pp. 51-58 } \\
\text { Khan and Parra (2003), pp. 264-266 } \\
\text { Lang (1998), pp. } 267\end{array}$ & $\begin{array}{l}\text { HubCo, Pakistan } \\
\text { Meghnaghat Power } \\
\text { Project, Bangladesh } \\
\text { Meizhou Wan, China } \\
\text { Pat Gen, Pakistan }\end{array}$ \\
\hline $\begin{array}{l}\text { Credit } \\
\text { Enhancement } \\
\text { Factors }\end{array}$ & $\begin{array}{l}\text { Chowdhury and Charoenngam (2008), pp. 267-283 } \\
\text { Khan and Parra (2003), pp. 254-264 } \\
\text { Gatti (2008), pp. 292-295 }\end{array}$ & $\begin{array}{l}\text { Dabhol Power, India } \\
\text { HubCo, Pakistan } \\
\text { Paiton 1, Indonesia }\end{array}$ \\
\hline
\end{tabular}

\subsection{Literature review}

A comprehensive collection of literature regarding PPP projects has been carried out while focusing mainly on the structure of SPV, legal and financial agreements, relevant laws, guidelines, standard of practice and support mechanism. It is found that different types of public-private partnerships (PPPs) have been practiced in worldwide infrastructure development (Davis 2003; Blackwell 2000). Though many PPP models exist, one thing is common and most essential in all these schemes - Special Purpose Vehicle (SPV). Creation of SPV or Project Company is the seminal step in any PPP infrastructure project. The International Project Finance Association (IPFA) posits that "at the heart of project finance transaction is the concession company, a Special Purpose Vehicle (SPV)". The competence of the SPV depends on the overall resources and capabilities of the constituent companies, its ability to formulate competitive financial and technical packages, and the partnering skills of the proposed project participants. 
The private-sector partner must have the necessary skills, experience and resources to manage a broad range of risks associated with long-term PPP. Zhang (2004) mentions that the selection of the right private-sector partner is a critical success factor for PPPs. It is needless to say that the SPV is formed through the participation of the privatesector. Chowdhury and Charoenngam (2008) have compared various SPV models used in many worldwide PPP projects. A qualitative research is done by Chowdhury and Chen (2010) to find the trends and techniques of SPV for Asia and Mediterranean Middle East. Chowdhury and Charoenngam (2009) in another research have identified the factors influencing finance of independent power producer (IPP) projects in Asia. Tiong and Alum (1997) analyzed the elements of selecting suitable concessionaire. Similarly, Zhang (2004) has studied the concessionaire selection practice in Hong Kong. Kumaraswsamy and Zhang (2001) have done research on governmental role in PPP particularly BOT-let infrastructure projects. Fishbein and Babbar (1996), Dailami and Leipziger (1997), Irwin et al. (1997), Klein (1997), and Ye and Tiong (2000) indicate that support from government plays significant role in PPP project success. Thus, various articles and useful knowledge are explored from online databases, journals, books and World Wide Web pages.

\subsection{Investigation of cases}

Cases on power generation projects in India, Pakistan, China, Thailand, Bangladesh, Singapore, Taiwan and Vietnam are investigated to find out the financial and legal factors for establishing SPV in Asia. These include Dabhol Independent Power Project (IPP) project in India, AES Lal Pir, Pat Gen and HubCo IPP projects in Pakistan, Laibin B, Shajiao C, Meizhou Wan and Huaibei Power Project in China, Meghnaghat power project in Bangladesh, PT Paiton Energy (Paiton 1) power project in Indonesia, BLCP Power project in Thailand, and Casecnan Water and Energy Project in Philippines.

\subsection{Questionnaire survey}

The designed questionnaire comprises a total of 75 factors (contractual: 16; enabling environment: 15; guarantees: 17 ; and credit enhancement: 27) for the set up of SPV for PPP power projects. These factors are gathered through comprehensive study of literature and from the above mentioned cases (as shown in Table 1). The authors have conducted a questionnaire survey from October 2009 to January 2010. The aim of the survey is to consolidate knowledge and expertise that would contribute to identifying the important factors for setting up SPV and to know the practices widely used in structuring SPV for PPP power projects in Asia. Respondents were asked to rate the importance/criticality of each factors on a five-point Likert rating scale ranging from 1 (least important) to 5 (most important). The questionnaire is divided into three parts (1) financial part; (2) legal part; and (3) credit enhancement. To remove any ambiguities in the questionnaire, a pilot test was carried out. Minor amendments were made accordingly to the results of the pilot test and a glossary was attached to reduce confusion and to refresh the memory of the respondents prior to mailing the questionnaire to a selected academic or professional in Asia. Table 2 shows the respondents' list of each country. 
Table 2. Countrywide respondents' details

\begin{tabular}{ccc}
\hline Country & Number of Respondents & Type of Organization \\
\hline Bangladesh & 4 & $\begin{array}{c}\text { Public: } 3 \\
\text { Private: } 1\end{array}$ \\
\hline Taiwan & 3 & $\begin{array}{c}\text { Public: } 1 \\
\text { Private: } 2\end{array}$ \\
\hline India & 4 & $\begin{array}{c}\text { Public: } 2 \\
\text { Private: } 2\end{array}$ \\
\hline Pakistan & 3 & Private: 3 \\
\hline Thailand & 6 & Public: 2 \\
& & Private: 4 \\
\hline Singapore & 5 & Public: 1 \\
& & Private: 4 \\
\hline Malaysia & 4 & Public: 2 \\
& & Private: 2 \\
\hline Vietnam & 2 & Private: 2 \\
\hline & Total 31 Public: 11 & \\
\hline
\end{tabular}

31 (out of 85) respondents have returned completed questionnaires from various different organizations in countries from Bangladesh, India, Pakistan, Thailand, Taiwan, China, Singapore, Malaysia and Vietnam. According to the central limit theorem, the relatively small sample (here, $\mathrm{n}=31$ ) is still valid for normal distribution (Cooper, Schindler 2003). All these respondents were involved in PPP projects and/or have done research in this area and have experience of 10 years or more. Some respondents were from organizations that have rich experience and knowledge in PPP such as Infrastructure Investment Facilitation Center (IIFC, Bangladesh), Infrastructure Development Company Limited (IDCOL, Bangladesh), Gammon Construction (Singapore), Hogan Lovels Lee and Lee (Singapore), Plenary Group (Singapore), 3PU in Malaysia, Commonwealth Secretariat Advisory Unit, and the World Bank. In other words, the respondents were lawyers, consultants, academic experts, financiers, contractors or from public clients.

\section{Data analysis}

\subsection{Contractual foundation benchmark}

\subsubsection{Off-take agreement}

Although responses vary from one respondent to another, they typically tend to center around a single value which can be deemed to be "representative" of criticality. Key statistics used are mean, standard deviation, ranking and percentage of responses per factor. Table 3 shows a summary of the responses. 
Table 3. Survey results

\begin{tabular}{|c|c|c|c|c|c|c|c|c|c|c|}
\hline \multirow{2}{*}{$\begin{array}{l}\text { Factors of Special Purpose } \\
\text { Vehicle for PPP Power Projects }\end{array}$} & \multirow{2}{*}{ Min } & \multirow{2}{*}{ Max } & \multirow{2}{*}{ Mean } & \multirow{2}{*}{$\mathrm{SD}$} & \multirow{2}{*}{ Rank } & \multicolumn{5}{|c|}{$\%$ of response per factor } \\
\hline & & & & & & 1 & 2 & 3 & 4 & 5 \\
\hline
\end{tabular}

\section{Contractual Foundation Benchmark: 16 factors}

\begin{tabular}{|c|c|c|c|c|c|c|c|c|c|c|}
\hline \multicolumn{11}{|c|}{ Off-take ( 7 factors) } \\
\hline Take or Pay off-take agreement & 2 & 5 & 3.83 & 0.93 & 13 & 0 & 6.4 & 32.3 & 32.3 & 29 \\
\hline $\begin{array}{l}\text { Hell or High Water off-take } \\
\text { agreement }\end{array}$ & 1 & 5 & 3.03 & 1.13 & 72 & 9.7 & 19.4 & 41.9 & 16.1 & 12.9 \\
\hline $\begin{array}{l}\text { SPV is obliged to make certain } \\
\text { payments to compensate the } \\
\text { off-taker, if it fails to meet its } \\
\text { delivery obligation }\end{array}$ & 1 & 5 & 3.45 & 0.92 & 58 & 3.2 & 9.7 & 35.5 & 41.9 & 9.7 \\
\hline $\begin{array}{l}\text { If off-taker does not dispatch } \\
\text { the plant, it makes a capacity } \\
\text { payment, but not energy payment }\end{array}$ & 2 & 5 & 3.25 & 0.93 & 69 & 0 & 22.6 & 38.7 & 29 & 9.7 \\
\hline $\begin{array}{l}\text { Guarantee from the off-taker } \\
\text { to indemnify SPV for all costs/ } \\
\text { losses incurred converting } \\
\text { payments from local currency to } \\
\text { foreign currency }\end{array}$ & 2 & 5 & 3.32 & 0.83 & 66 & 0 & 16.1 & 41.9 & 35.5 & 6.5 \\
\hline Merchant Facility & 1 & 4 & 2.71 & 0.93 & 73 & 9.7 & 32.3 & 35.5 & 22.5 & 0 \\
\hline $\begin{array}{l}\text { Provision of third party sales in } \\
\text { case of default of off-taker }\end{array}$ & 2 & 5 & 3.58 & 1.08 & 44 & 0 & 19.4 & 29 & 25.8 & 25.8 \\
\hline
\end{tabular}

\begin{tabular}{|c|c|c|c|c|c|c|c|c|c|c|}
\hline \multicolumn{11}{|c|}{ Supply (4 factors) } \\
\hline $\begin{array}{l}\text { Long term‘ Supply or Pay’ } \\
\text { agreement }\end{array}$ & 2 & 5 & 3.80 & 0.833 & 14 & 0 & 3.2 & 35.5 & 38.7 & 22.6 \\
\hline $\begin{array}{l}\text { Long term 'Supply and Pay' } \\
\text { agreement }\end{array}$ & 1 & 5 & 3.22 & 1.02 & 70 & 3.2 & 19.4 & 41.9 & 22.6 & 12.9 \\
\hline Spot Purchase & 1 & 5 & 2.61 & 0.88 & 75 & 6.5 & 41.9 & 38.7 & 9.7 & 3.2 \\
\hline $\begin{array}{l}\text { Secure certain amount of energy } \\
\text { through long term supply } \\
\text { agreement }\end{array}$ & 2 & 5 & 3.87 & 0.92 & 10 & 0 & 9.7 & 19.3 & 45.2 & 25.8 \\
\hline
\end{tabular}

\begin{tabular}{llllllllllll}
\hline \multicolumn{11}{c}{ Counter Parties (5 factors) } \\
\hline Credit quality of the off-taker & 2 & 5 & 4.13 & 0.92 & 4 & 0 & 6.5 & 16.1 & 35.5 & 41.9 \\
\hline Credit quality of the supplier & 3 & 5 & 4.30 & 0.74 & 1 & 0 & 0 & 16.1 & 38.7 & 45.2 \\
\hline $\begin{array}{l}\text { Rated O\&M contract with } \\
\text { performance damage }\end{array}$ & 2 & 5 & 3.58 & 0.84 & 42 & 0 & 6.5 & 45.2 & 32.3 & 16.1 \\
\hline $\begin{array}{l}\text { Country's history on } \\
\text { international borrowing records }\end{array}$ & 2 & 5 & 3.45 & 0.88 & 56 & 0 & 12.9 & 41.9 & 32.3 & 12.9 \\
\hline $\begin{array}{l}\text { Credit quality of the sponsor } \\
\text { particularly an EPC contractor }\end{array}$ & 2 & 5 & 3.51 & 0.85 & 51 & 0 & 9.7 & 41.9 & 35.5 & 12.9 \\
\hline
\end{tabular}

\section{Enabling Environmental Benchmark : 15 factors}

Policy and Regulatory Framework (10 factors)

\begin{tabular}{lllllllllll}
\hline Long track record of country's & 2 & 5 & 3.90 & 0.74 & 7 & 0 & 3.2 & 22.6 & 54.8 & 19.4
\end{tabular}

legal stability 
Continue of Table 3

\begin{tabular}{|c|c|c|c|c|c|c|c|c|c|c|}
\hline \multirow{2}{*}{$\begin{array}{l}\text { Factors of Special Purpose } \\
\text { Vehicle for PPP Power Projects }\end{array}$} & \multirow{2}{*}{ Min } & \multirow{2}{*}{ Max } & \multirow{2}{*}{ Mean } & \multirow{2}{*}{$\mathrm{SD}$} & \multirow{2}{*}{ Rank } & \multicolumn{5}{|c|}{$\%$ of response per factor } \\
\hline & & & & & & 1 & 2 & 3 & 4 & 5 \\
\hline $\begin{array}{l}\text { Established government's } \\
\text { template for infrastructure } \\
\text { financing }\end{array}$ & 2 & 5 & 3.80 & 0.87 & 15 & 0 & 6.5 & 29 & 41.9 & 22.6 \\
\hline $\begin{array}{l}\text { Comprehensive and transparent } \\
\text { concession law }\end{array}$ & 3 & 5 & 4.25 & 0.68 & 2 & 0 & 0 & 12.9 & 48.4 & 38.7 \\
\hline Competitive bidding award & 2 & 5 & 3.70 & 0.90 & 28 & 0 & 6.4 & 38.7 & 32.32 & 22.6 \\
\hline $\begin{array}{l}\text { Strong political commitment } \\
\text { (Clear Objective, Dispute } \\
\text { Resolution) }\end{array}$ & 2 & 5 & 4.25 & 0.85 & 3 & 0 & 6.4 & 6.4 & 41.9 & 45.2 \\
\hline $\begin{array}{l}\text { Institutional environment } \\
\text { (such as skill and dedicated } \\
\text { Government staff to oversee } \\
\text { design and implementation } \\
\text { of PPP) }\end{array}$ & 2 & 5 & 3.80 & 1.07 & 17 & 0 & 16 & 19.4 & 32.3 & 32.3 \\
\hline $\begin{array}{l}\text { Government issuance of policy } \\
\text { guideline }\end{array}$ & 2 & 5 & 3.77 & 0.88 & 15 & 0 & 6.4 & 32.3 & 38.7 & 22.6 \\
\hline $\begin{array}{l}\text { Well developed legal system and } \\
\text { significant precedent exists }\end{array}$ & 3 & 5 & 3.90 & 0.65 & 6 & 0 & 0 & 25.8 & 58.1 & 16.1 \\
\hline Legal statutes for project finance & 2 & 5 & 3.90 & 0.90 & 8 & 0 & 6.5 & 25.8 & 38.7 & 29 \\
\hline $\begin{array}{l}\text { Trade liberalization, Privatization } \\
\text { or deregulation of key sectors } \\
\text { (such as electricity, utilities etc.) }\end{array}$ & 1 & 5 & 3.61 & 1.02 & 40 & 3.2 & 6.5 & 38.7 & 29 & 22.6 \\
\hline
\end{tabular}

\begin{tabular}{|c|c|c|c|c|c|c|c|c|c|c|}
\hline \multicolumn{11}{|c|}{ Implementation Agreement (5 factors) } \\
\hline $\begin{array}{l}\text { Government allows dispute } \\
\text { resolution in international } \\
\text { arbitration }\end{array}$ & 2 & 5 & 3.77 & 0.88 & 19 & 0 & 9.7 & 22.6 & 48.4 & 19.4 \\
\hline $\begin{array}{l}\text { No significant instance of } \\
\text { expropriation, currency } \\
\text { inconvertibility or contract } \\
\text { abrogation by the government }\end{array}$ & 2 & 5 & 3.71 & 0.97 & 27 & 0 & 9.7 & 35.5 & 29 & 25.8 \\
\hline $\begin{array}{l}\text { Government to carry out all } \\
\text { preparatory work including land } \\
\text { acquisition and utility removal } \\
\text { at its cost }\end{array}$ & 1 & 5 & 3.42 & 0.92 & 60 & 3.2 & 9.7 & 38.7 & 38.7 & 9.7 \\
\hline $\begin{array}{l}\text { Concession Agreement, off-take } \\
\text { and fuel supply agreement are } \\
\text { in one agreement i.e "all in one } \\
\text { package" }\end{array}$ & 2 & 5 & 3.51 & 0.82 & 50 & 0 & 12.9 & 32.3 & 45.2 & 9.7 \\
\hline $\begin{array}{l}\text { De-linking PPA and fuel Supply } \\
\text { Agreement }\end{array}$ & 1 & 4 & 3.38 & 0.71 & 62 & 3.2 & 3.2 & 45.2 & 48.4 & 0 \\
\hline
\end{tabular}

Agreement

Guarantee Benchmark : 17 factors

\begin{tabular}{lllllllllll}
\hline \multicolumn{11}{c}{ Government (11 factors) } \\
\hline Government's guarantee on debt & 1 & 5 & 3.48 & 1.12 & 55 & 6.5 & 12.9 & 22.6 & 41.9 & 16.1 \\
\hline
\end{tabular}


Continue of Table 3

\begin{tabular}{|c|c|c|c|c|c|c|c|c|c|c|}
\hline \multirow{2}{*}{$\begin{array}{l}\text { Factors of Special Purpose } \\
\text { Vehicle for PPP Power Projects }\end{array}$} & \multirow{2}{*}{ Min } & \multirow{2}{*}{ Max } & \multirow{2}{*}{ Mean } & \multirow{2}{*}{ SD } & \multirow{2}{*}{ Rank } & \multicolumn{5}{|c|}{$\%$ of response per factor } \\
\hline & & & & & & 1 & 2 & 3 & 4 & 5 \\
\hline $\begin{array}{l}\text { Limited counter guarantee } \\
\text { in monetary value by the } \\
\text { government on behalf of the } \\
\text { off-taker }\end{array}$ & 2 & 5 & 3.51 & 0.81 & 48 & 0 & 9.7 & 38.7 & 41.9 & 9.7 \\
\hline $\begin{array}{l}\text { Support letter from the host } \\
\text { government which causes } \\
\text { off-taker to discharge its } \\
\text { payment obligation }\end{array}$ & 1 & 5 & 3.61 & 0.92 & 38 & 25.8 & 35.5 & 22.6 & 16.2 & 0 \\
\hline $\begin{array}{l}\text { Price regulation guarantee on } \\
\text { market by the host government }\end{array}$ & 2 & 5 & 4.0 & 0.89 & 5 & 0 & 3.2 & 29 & 32.3 & 35.5 \\
\hline $\begin{array}{l}\text { Government's guarantee } \\
\text { on supplier's performance } \\
\text { (if supplier is State Owned } \\
\text { Enterprise) }\end{array}$ & 2 & 5 & 3.71 & 0.90 & 26 & 0 & 9.7 & 29 & 41.9 & 19.4 \\
\hline $\begin{array}{l}\text { Government's counter guarantee } \\
\text { on payment (if off-taker is SOE) }\end{array}$ & 2 & 5 & 3.71 & 0.78 & 23 & 0 & 3.2 & 38.7 & 41.9 & 16.1 \\
\hline $\begin{array}{l}\text { Agreement that protects SPV } \\
\text { from increases in country taxes } \\
\text { or a change in country law }\end{array}$ & 2 & 5 & 3.67 & 0.90 & 29 & 0 & 9.7 & 32.3 & 38.7 & 19.3 \\
\hline $\begin{array}{l}\text { Force majeure event by } \\
\text { government is 'deemed } \\
\text { dispatched' and oblige to make } \\
\text { capacity payment to SPV }\end{array}$ & 2 & 5 & 3.87 & 0.95 & 11 & 0 & 6.4 & 32.3 & 29 & 32.3 \\
\hline $\begin{array}{l}\text { Use of stabilization clause } \\
\text { or stabilization guarantees in } \\
\text { the contract between the host } \\
\text { government and SPV }\end{array}$ & 2 & 5 & 3.67 & 0.90 & 29 & 0 & 9.7 & 32.3 & 38.7 & 19.3 \\
\hline $\begin{array}{l}\text { Government's commitment to } \\
\text { renegotiate tariff in the event } \\
\text { of currency devaluation }\end{array}$ & 2 & 5 & 3.64 & 0.79 & 33 & 0 & 3.2 & 45.2 & 35.5 & 16.1 \\
\hline $\begin{array}{l}\text { Unconditional and irrevocable } \\
\text { guarantee by the host } \\
\text { government }\end{array}$ & 1 & 5 & 3.58 & 1.14 & 45 & 6.4 & 12.9 & 16.1 & 45.2 & 19.4 \\
\hline
\end{tabular}

government

\begin{tabular}{lccccccccccc}
\hline & \multicolumn{1}{c}{ Others (6 factors) } & & & & & & \\
\hline $\begin{array}{l}\text { Central bank's guarantee } \\
\text { on Currency Availability, } \\
\text { Convertibility and Transferability }\end{array}$ & 2 & 5 & 3.64 & 0.83 & 34 & 0 & 9.7 & 29 & 48.4 & 12.9 \\
\hline $\begin{array}{l}\text { Multi-lateral Development } \\
\text { Banks' credit guarantee }\end{array}$ & 2 & 5 & 3.67 & 0.94 & 31 & 0 & 6.4 & 45.2 & 22.6 & 25.8 \\
\hline $\begin{array}{l}\text { Export Credit Agencies credit } \\
\text { guarantee }\end{array}$ & 2 & 5 & 3.30 & 0.86 & 67 & 0 & 16.1 & 48.4 & 25.8 & 9.7 \\
\hline $\begin{array}{l}\text { Credit facilities by the } \\
\text { commercial banks }\end{array}$ & 2 & 5 & 3.77 & 0.76 & 18 & 0 & 3.2 & 32.3 & 48.4 & 16.1 \\
\hline $\begin{array}{l}\text { Letter of credit from a bank to } \\
\text { tide over temporary liquidity } \\
\text { mismatches on behalf of } \\
\text { off-taker }\end{array}$ & 2 & 5 & 3.61 & 0.72 & 37 & 0 & 3.2 & 45.2 & 38.7 & 12.9 \\
\hline
\end{tabular}


Continue of Table 3

\begin{tabular}{lcccccccccc}
\hline $\begin{array}{l}\text { Factors of Special Purpose } \\
\text { Vehicle for PPP Power Projects }\end{array}$ & Min & Max & Mean & SD & Rank & \multicolumn{5}{c}{ \% of response per factor } \\
\hline $\begin{array}{l}\text { Off-taker's guarantee for } \\
\text { payment (Letter of credit from } \\
\text { the off-taker) }\end{array}$ & 2 & 5 & 3.54 & 0.80 & 47 & 0 & 6.5 & 45.2 & 35.5 & 12.9 \\
\hline
\end{tabular}

\begin{tabular}{|c|c|c|c|c|c|c|c|c|c|c|}
\hline \multicolumn{11}{|c|}{ Credit Enhancement Benchmark : 27 factors } \\
\hline \multicolumn{11}{|c|}{ Shareholders (6 factors) } \\
\hline $\begin{array}{l}\text { Contingent Equity support by } \\
\text { the sponsors }\end{array}$ & 2 & 5 & 3.87 & 0.84 & 9 & 0 & 6.5 & 22.6 & 48.4 & 22.6 \\
\hline $\begin{array}{l}\text { Standby credit guarantee by } \\
\text { the sponsors }\end{array}$ & 2 & 5 & 3.51 & 0.85 & 51 & 0 & 12.8 & 32.3 & 45.2 & 9.7 \\
\hline $\begin{array}{l}\text { Shareholder's retention } \\
\text { agreement }\end{array}$ & 2 & 5 & 3.71 & 0.82 & 24 & 0 & 6.4 & 32.3 & 45.2 & 16.1 \\
\hline $\begin{array}{l}\text { Ability to Exit through sales } \\
\text { of shares from SPV }\end{array}$ & 1 & 5 & 3.06 & 1.03 & 71 & 6.5 & 25.8 & 25.8 & 38.7 & 3.2 \\
\hline $\begin{array}{l}\text { Shareholders' agreement that } \\
\text { SPV reserves a maintenance } \\
\text { account for O\&M before making } \\
\text { any distribution to shareholders }\end{array}$ & 2 & 5 & 3.83 & 0.73 & 12 & 0 & 3.2 & 25.8 & 54.9 & 16.1 \\
\hline $\begin{array}{l}\text { Claw back guarantee by the } \\
\text { project sponsors and passive } \\
\text { equity investors }\end{array}$ & 2 & 5 & 3.42 & 0.76 & 59 & 0 & 6.5 & 54.8 & 29 & 9.7 \\
\hline
\end{tabular}

\begin{tabular}{|c|c|c|c|c|c|c|c|c|c|c|}
\hline \multicolumn{11}{|c|}{ Government (5 factors) } \\
\hline $\begin{array}{l}\text { Letter of Credit by the host } \\
\text { government }\end{array}$ & 2 & 5 & 3.71 & 0.82 & 24 & 0 & 6.4 & 32.3 & 45.2 & 16.1 \\
\hline $\begin{array}{l}\text { Establishment of government } \\
\text { funded debt reserve account if } \\
\text { state-owned off-taker is unable } \\
\text { to make necessary payments } \\
\text { to the lenders }\end{array}$ & 1 & 5 & 3.67 & 0.94 & 31 & 3.2 & 3.2 & 35.5 & 38.7 & 19.4 \\
\hline $\begin{array}{l}\text { Presence of host government } \\
\text { grants }\end{array}$ & 1 & 5 & 3.51 & 1.09 & 54 & 3.2 & 12.9 & 35.5 & 25.8 & 22.6 \\
\hline $\begin{array}{l}\text { Presence of Subordinated Debt } \\
\text { (by Host Government) }\end{array}$ & 1 & 5 & 3.42 & 0.92 & 60 & 3.2 & 9.7 & 38.7 & 38.7 & 9.7 \\
\hline $\begin{array}{l}\text { Presence of Equity from } \\
\text { Government/ Government } \\
\text { Agency }\end{array}$ & 1 & 5 & 3.38 & 1.05 & 63 & 6.4 & 12.9 & 25.8 & 45.2 & 9.7 \\
\hline
\end{tabular}

\begin{tabular}{|c|c|c|c|c|c|c|c|c|c|c|}
\hline \multicolumn{11}{|c|}{ Third Parties (8 factors) } \\
\hline $\begin{array}{l}\text { Involvement of Multilateral } \\
\text { Agencies }\end{array}$ & 2 & 5 & 3.51 & 0.81 & 48 & 0 & 6.5 & 48.4 & 32.3 & 12.9 \\
\hline $\begin{array}{l}\text { Involvement of Export Credit } \\
\text { Agencies }\end{array}$ & 2 & 5 & 3.45 & 0.88 & 56 & 0 & 12.9 & 41.9 & 32.3 & 12.9 \\
\hline Involvement of security trustee & 2 & 5 & 3.61 & 1.02 & 40 & 0 & 16.1 & 29 & 32.3 & 22.6 \\
\hline $\begin{array}{l}\text { Involvement of insurance } \\
\text { companies (Business interruption } \\
\text { and casualty insurance policies } \\
\text { in place) }\end{array}$ & 2 & 5 & 3.64 & 0.91 & 36 & 0 & 6.4 & 45.2 & 25.8 & 22.6 \\
\hline
\end{tabular}


End of Table 3

\begin{tabular}{|c|c|c|c|c|c|c|c|c|c|c|}
\hline \multirow{2}{*}{$\begin{array}{l}\text { Factors of Special Purpose } \\
\text { Vehicle for PPP Power Projects }\end{array}$} & \multirow{2}{*}{ Min } & \multirow{2}{*}{ Max } & \multirow{2}{*}{ Mean } & \multirow{2}{*}{ SD } & \multirow{2}{*}{ Rank } & \multicolumn{5}{|c|}{$\%$ of response per factor } \\
\hline & & & & & & 1 & 2 & 3 & 4 & 5 \\
\hline $\begin{array}{l}\text { Presence of Subordinated Debt } \\
\text { (by Multilateral Agency) }\end{array}$ & 1 & 5 & 3.25 & 0.81 & 60 & 3.2 & 6.5 & 58.1 & 25.8 & 6.4 \\
\hline Commercial Paper from Banks & 1 & 4 & 2.61 & 0.80 & 74 & 0 & 16.1 & 48.4 & 25.8 & 9.7 \\
\hline $\begin{array}{l}\text { Establishment of specialized } \\
\text { intermediary (such as } \\
\text { Infrastructure Development } \\
\text { Finance Company etc) with } \\
\text { equity participation from } \\
\text { government, domestic financial } \\
\text { institutions. }\end{array}$ & 2 & 5 & 3.54 & 0.72 & 46 & 0 & 6.5 & 38.7 & 48.3 & 6.5 \\
\hline $\begin{array}{l}\text { Financing with political risk } \\
\text { insurance from Multi-lateral } \\
\text { agencies or Export Credit } \\
\text { agencies or insurance companies }\end{array}$ & 2 & 5 & 3.80 & 0.87 & 15 & 0 & 9.7 & 19.4 & 51.6 & 19.4 \\
\hline
\end{tabular}

Capital Structure Mechanism (8 factors)

\begin{tabular}{lllllllllll}
\hline Creation of Debt Service & 2 & 5 & 3.74 & 0.81 & 22 & 0 & 3.2 & 38.7 & 38.7 & 19.4
\end{tabular}

Reserve Fund

\begin{tabular}{lllllllllll}
\hline $\begin{array}{l}\text { A trust that grants SPV a priority } \\
\text { interest in portion of off-taker's } \\
\text { cash collection in case the } \\
\text { off-taker defaults in payment } \\
\text { obligation }\end{array}$ & 2 & 4 & 3.35 & 0.66 & 64 & 0 & 6.5 & 54.8 & 35.5 & 3.2 \\
\hline
\end{tabular}

\begin{tabular}{lllllllllll}
\hline $\begin{array}{l}\text { Standby letter of credit backing } \\
\begin{array}{l}\text { Contractor's performance to } \\
\text { fulfill its obligation }\end{array}\end{array}$ & 2 & 5 & 3.58 & 0.84 & 42 & 0 & 9.7 & 35.5 & 41.9 & 12.9 \\
\hline
\end{tabular}

$\begin{array}{llllll}\text { Senior lender's acceptance of } & 2 & 5 & 3.61 & 0.95 & 39\end{array}$

back-ended payment profile (i.e.

flexible repayment schedule)

\begin{tabular}{|c|c|c|c|c|c|c|c|c|c|c|}
\hline $\begin{array}{l}\text { A subordination agreement } \\
\text { among government, SPV and } \\
\text { lenders for short term cash flow } \\
\text { pressure }\end{array}$ & 2 & 5 & 3.35 & 0.80 & 65 & 0 & 12.9 & 45.2 & 35.5 & 6.4 \\
\hline $\begin{array}{l}\text { Indexation formula that adjusts } \\
\text { the local currency tariffs for } \\
\text { inflation and changes in tax }\end{array}$ & 2 & 5 & 3.64 & 0.87 & 35 & 0 & 9.7 & 32.3 & 41.9 & 16.1 \\
\hline $\begin{array}{l}\text { Establish an escrow agreement } \\
\text { between SPV and off-taker to } \\
\text { capture revenues from off-taker } \\
\text { customer to support off-taker's } \\
\text { payment obligation }\end{array}$ & 2 & 5 & 3.77 & 0.88 & 19 & 0 & 6.4 & 32.3 & 38.7 & 22.6 \\
\hline $\begin{array}{l}\text { Establish a lender managed } \\
\text { escrow account for deposit } \\
\text { revenues }\end{array}$ & 2 & 5 & 3.51 & 0.92 & 53 & 0 & 12.9 & 38.7 & 32.3 & 16.1 \\
\hline
\end{tabular}


From the literature review and case investigation, it is found that two off-take agreements are widely used: (1) take or pay and (2) hell or high water. In the questionnaire survey, respondents were asked to indicate which of these agreements they would like to recommend. It is seen that "take or pay" off-take agreement is mostly recommended by the respondents. (Note: The high mean ratios for "take or pay" and "hell or high water" are 3.83 and 3.03, respectively.) Though the "hell or high water" off-take agreement contributes high indirect credit enhancement to the lenders, but it needs to be projected carefully due to the reason that the project is capable enough to produce its utility. It is interesting to find that "hell or high water" has high standard deviation which signifies that the data are spread over a large range of values. The rank of this factor is 72 whereas the rank of 'take or pay' is only 13. From the analysis, it is also seen that the culture of merchant facility (here the mean is 2.71) is not yet a popular practice in the emerging market of Asia. The rank of this factor is 73. It is also noteworthy that none of the respondents gave maximum value (i.e. 5 = extremely important) to this factor. Thus, it signifies that the respondents preferred long term off-take contract to reduce market risks which are prominent in nature in Asia. It is also noted that the respondents made a moderate to high rating on "provision of third party sales in case of default of off-taker" with a mean of 3.58 .

\subsubsection{Supply agreement}

Among the three types of supply agreement (i.e. long term 'supply or pay'; long term 'supply and pay' and, spot purchase), the most preferred agreement by the respondents is long term "supply or pay" (as the mean is 3.80 and std. dev. is 0.83 ). A closer examination on supply agreements between "supply or pay" and "supply and pay" reveals that the respondents, in general, rated the latter lower than the former. This observation again reinforces the assertion that respondents prefer long time "supply or pay" agreement with a quality credit supplier (here, the mean is 4.3 and std. dev. is 0.74 ). This type of agreement gives the SPV a maximum degree of flexibility as long as yearly minimum purchase requirement is maintained. Moreover, the SPV can buy fuels from other suppliers if the original supplier fails to deliver or the fuel is off-spec and the supplier pays the liquidated damage to SPV to buy on-spec fuel in the open market. The analysis shows that spot purchase got the lowest mean value of 2.61 and ranked at the last (i.e. 75) by the respondents. This may be due to the fact that the respondents have considered that in this region (i.e. in Asia), there are limited numbers of supplier and chances of raw material price escalation are high.

\subsubsection{Counter parties}

Very high scores are obtained by "credit quality of supplier" and "credit quality of off-taker". $45.2 \%$ and $41.9 \%$ of the respondents gave highest rating (i.e. $5=$ extremely important) on these two factors respectively. Credit quality of supplier and credit quality of off-taker have ranked $1^{\text {st }}$ and $4^{\text {th }}$ among all 75 factors in this analysis. The respondents have significantly considered this issue as they are concerned that a project may get downgraded due to the poor financial condition of the off-taker and supplier. "rated O\&M contract with performance damage" is being accounted as another dominant fac- 
tor (i.e. mean 3.58 and std. dev. 0.84 ) in considering credit quality of counter parties. The sponsor also can become a source of counter-party risk if it fails to provide equity in the construction stage of a project. This issue is also being reflected in the questionnaire survey of the respondents as it is seen that "credit quality of EPC contractor" also achieved higher mean (i.e. 3.51). A creditworthy contractor with a fixed price and certain- dates turnkey contract is able to bear completion risks. The experiences in Asia have shown that counterparty's weak underlying economy and financial mismanagement can put the SPV into financial difficulty or even lead to restructuring of PPP to survive in the market.

\subsection{Enabling environment benchmark}

\subsubsection{Policy and regulatory framework}

Among the ten legal and regulatory factors of this group, the majority of respondents considered "comprehensive and transparent law", and "strong political commitment" are the most important factors (with a mean of 4.25). Though these two factors have same mean but comprehensive and transparent law has achieved higher ranking due to its lower standard deviation than that of strong political commitment (as shown in Table 3). Factors "well developed legal system" and "long track record of country's legal stability" are ranked $6^{\text {th }}$ and $15^{\text {th }}$. It is noteworthy that $54.8 \%$ of the respondents gave higher weightage (i.e. 4 = very important) to "long track record of country's stability". The lowest score gained in this group is by "trade liberalization, privatization or deregulation of key sectors" whose mean is also considerably higher (i.e. 3.61), comparing the mean of other factors of another groups. It is also interesting to see that all the ten factors have scored a mean higher than 3.60. This indicates that the respondents have considered the factors of this group with significant importance for setting up SPV of PPP projects. The mean score of this group is 3.88 , which is much higher than any mean score of the contractual benchmark factors or guarantee benchmark factors. This implies that most of the respondents admitted the importance of legal and institutional framework factors as a cornerstone for setting up SPV in Asian countries.

\subsubsection{Implementation agreement}

From the analysis, the most dominant factor considered by the respondents in implementation agreement group is "government's allowance in dispute resolution in international arbitration". It is thus inferred that respondents have profoundly considered dispute resolution mechanisms (such as arbitration and mediation) for overseas cases, especially when there is any suspicion that the courts of the host country will not treat a foreign investor fairly. The waiver of sovereign immunity converts the government into a commercial party for purposes of resolving legal issues. The respondents also considered "expropriation, currency inconvertibility or contract abrogation by host government" an important legal factor which has a mean 3.71 followed by the former (i.e. 3.77). A closer examination on concession agreement between "de-linking PPA and fuel supply" and "all in one package" reveals that the respondents made higher rating to the latter one (i.e. 3.51). De-linking Power Purchase Agreement (PPA) and supply 
agreement have attained a mean score of 3.38 which indicates that the respondents did not prefer/prioritize this mechanism as an influencing factor for setting up SPV. Though developers are considering PPA and fuel supply agreements separately nowadays, it is seen in many IPP projects in Mexico (Davis 2003). This may due to the fact that if PPA and fuel supply agreement are de-linked, sponsors would have to take market and price risk in emerging markets in Asia. Thus, the respondents preferred to have the concession agreement, the off-take agreement and/or input supply agreement in one package, which is reflected in the outcome of the questionnaire survey. To kick-off a project on time the factor "government to carry out land acquisition" has also got significant importance with a mean of 3.42. To accelerate project implementation, the government may, to some extent, furnish all these facilities to the private investors in advance. All these factors require due diligence before setting up SPV for PPP power projects.

\subsection{Guarantee benchmark}

\subsubsection{Government}

Among eleven types of government guarantees of this group, "price regulation guarantee on market by the host government" has achieved the highest mean (i.e. 4.0). The other high scored factor is "force majeure event by government is deemed dispatched and oblige to make capacity payment to SPV". It is to be noted that the risk of force majeure events, if unallocated away from the project, will limit most projects to the "BBB" category or below (Standard \& Poor's, Project and Infrastructure Finance Review 2001). It is also important to find out that the respondents made equal importance to "government's counter guarantee on off-taker's payment" and "government's counter guarantee on supplier's performance". Both these factors have achieved a high mean 3.71. This reveals that the respondents have considered these two factors equally important for setting up SPV. Though the experiences from India (i.e. Dabhol Project) and Indonesia (i.e. Paiton Project) show that counter guarantees from the host government can be unreliable, there is still a strong belief that these guarantee instruments have significant importance on decision making for setting up SPV of PPP power projects in Asia. Though $25.8 \%$ of the respondents gave not important (i.e. $0=$ not important) to "support letter from the government", yet it got a mean of 3.61 which is also high compared to other factors considered in the questionnaire. It reflects that support letter has significant importance, as it provides a statement of support and moral obligation from the highest level in the government.

\subsubsection{Third parties}

It is found that, the respondents have made highest rating to "credit facilities by the commercial banks" in this group. Other important factors in the group are "MDBs credit guarantee" and "central bank guarantee on currency convertibility, availability and transferability". The respondents have chosen this factor because it has immense impact on the structuring of SPV. This guarantee ensures the lenders against currency mismatch between revenues and the costs. This type of obligation from the bank helps SPV to make repatriation of profits and the servicing of foreign debts to its investors and lenders. 
Among two types of "letter of credit" which ensures payment guarantee by the SPV to the lenders, the analysis reveals that the respondents preferred "letter of credit from a bank to tide over temporary liquidity mismatches on behalf of off-taker" to "letter of credit from off-taker". This may be due to the fact that almost all the off-takers in this region are state-owned enterprises and their underlying economic condition are not sound enough to provide payment guarantees. Moreover, the market condition is so volatile that even a stable off-taker finds it difficult to make payment during some stages of project life-cycle. In a developing country, the political and economic situation as well as creditworthiness of an off-taker can change considerably in just a few years. Thus the higher mean (i.e. 3.61) reflects the respondents' prudent judgment to have letter of credit from bank on behalf of off-taker to tide over temporary liquidity mismatch during the course of dry market condition.

\subsection{Credit enhancement benchmark}

\subsubsection{Shareholder}

The strength of the shareholder is measured not only by its financial condition but also by its commitment to the project. These are measured by contingent equity support, standby credit support, completion guarantee and shareholders' interest on shares, and received distribution. A very high mean (i.e. 3.7) is achieved by "contingent equity support from sponsors" from the respondents. The rank of this factor is 9 . This is a commitment in lieu of completion guarantee and also a mitigation tool against abandonment risk to a project. This device is frequently used to provide the SPV and the lenders a degree of protection against certain perceived risks. The respondents have judged this factor with high priority because such commitment helps to share the burden of risks during the time of debt restructuring in many past PPP projects in Asia. The respondents gave a high weightage to "retention agreement" which has a mean of 3.71 and a standard deviation of 0.82 . This signifies that the respondents do not want a shareholder to exit from SPV where the relevant shareholder's obligations remain unperformed. The respondents may like to see continued presence within the SPV of the sponsor. The result is also reflected in "ability to exit" which has scored a mean of 3.06 and a standard deviation of 1.03. "Contingent equity support from the sponsors" and "project completion guarantee by the sponsors" have achieved the highest mean (i.e. 3.87) followed by "shareholder's agreement that SPV reserves a maintenance account" with a mean of 3.83 in this group. Moreover, a higher percentage of responses on greater weightage (i.e. $4=$ very important and $5=$ extremely important) are found for these factors as well.

\subsubsection{Government}

The length of concession period and degree of government involvement has significant influence on the financial structuring of SPV. Five factors fell into this group. With a mean of 3.71, "letter of credit by the government" has gained highest rank from the respondents. Other significant factor is "establishment of government funded debt reserve account for off-taker". This factor helps SPV with economic control on revenue stream which ensures lenders to get their debt service in case of off-taker's inability 
for payment. From the analysis, it is seen that the government's grant (mean 3.51 and standard deviation 1.09) is also a significant credit enhancement factor for a PPP power project in Asia. SPV can enhance project economics through government non-repaying grants and subordinated debt. Though the equity and debt guarantee from the government have higher impact on the ability to raise finance (Fishbein, Babbar 1996), but it is interesting to find out that the respondents in this questionnaire survey made higher rating on government grants. It reflects that the respondents prefer to use less government financial exposure considering among equity, debt and grant.

\subsubsection{Third parties}

The respondents made highest rating to "financing with political risk coverage from MDBs/ECAs" with a mean of 3.80 in this group. Some ECAs and MDBs provide guarantees or insurance of loans in relation to political events. $51.6 \%$ of respondents gave higher value (i.e. 4 = very important) to this factor. The rank of this factor is 15 . It is needless to mention that political turbulence and instability in some developing Asian countries is quite prominent. Examples are Thailand and Pakistan. Experiences from the past PPP power projects show that the governments make greater efforts to ensure loan repayments to MDBs or ECAs in case of political events. Therefore, the involvement of MDBs or ECAs with this type of loans helps SPV to make payment due to occurrence of political event and protects it from insolvency risk.

The sources of finance are also important factors in view of financing by the participants. The involvements of international institutions increase the confidence level of local commercial banks and may consequently reduce the interest rate of debt. This has been reflected in the questionnaire survey where MDB has scored a high mean of 3.51. It is interesting to see that the respondents gave a moderate rating to "MDBs subordinate debt". This may reveal that the respondents prefer MDBs' more participation in debt and guarantees (such as partial credit guarantee and partial risk guarantee) and their roles as a conduit for funding from other commercial banks to their participation on subordinated debt. It is true that the involvement of MDBs can help to raise local currency bonds, currency swaps to provide long term debt financing.

One of the significant credit enhancement factor used in many PPP projects in Asia is "establishment of specialized intermediary with equity participation from government and/or domestic financial institutions" such as Private Sector Energy Development Fund (PSEDF) of Pakistan or Private Sector Infrastructure Development Fund (PSIDF) of Bangladesh. $48.3 \%$ of respondents gave higher rating (i.e. $4=$ very important) to this factor. The analysis reveals that this factor got a moderate rating (i.e. within the range of important to very important) with a mean of 3.54 .

\subsubsection{Capital structure enhancement mechanism}

The SPV is required to maintain numerous bank accounts with funds earmarked for different purposes. Amongst them, one is debt service reserve fund (DSRF) which meets debt services in circumstances where there are insufficient funds in the revenue account. The respondents of the questionnaire survey have given high importance to this factor 
which is reflected from its obtained mean 3.74. On the other hand, escrow account is set up to prevent siphoning of the project's revenue for other purposes by sponsors. The SPV needed to ensure this account abided by the covenants of the lenders as they wanted to control the cash available for debt service (CADS) and free cash flow (FCF) of a project. The respondents made a moderate rating to "establish a lender managed escrow account for deposit revenue" which has a mean of 3.51 but they made a high rank (i.e. mean 3.77) to "establish an escrow agreement between SPV and off-taker to capture revenue from off-taker to support off-taker's payment obligation" which is different from lender managed escrow account. This is a kind of security package that ensures the SPV a priority interest in a portion of off-taker's operating cash collection in case of off-taker's default on payment obligation. For the reader's information, this kind of mechanism has been used in TermoEmcali power plant in Colombia.

Another interesting credit enhancement mechanism is 'senior lender's acceptance of back-ended repayment profile'. Senior lenders' acceptance to longer maturity is beneficial for SPV to structure its payment profile. The respondents of this questionnaire made a moderate to high rating (i.e. mean 3.61) to this factor as they might consider that the base case scenario is sometimes difficult to achieve in the Asian market.

\section{Discussion of findings}

Off-take and supply agreements are crucial agreements to decide on the magnitude of financing by the lenders as well as from SPV's point of view. These also become vital issues in some instances of failed PPP power projects in Asia. For example, in a recent study, it was found that Indian giant Tata Group had finally given up its US\$ 3 billion investment plan on fertilizer, power and steel projects in Bangladesh (Saleque 2009). This is due to the fact that the Bangladesh government was unable to give assurance for a long term gas supply. Khan and Parra (2003: 113-114) stated that a project that has an off-take agreement is always more attractive than one that does not. The market risks particularly in Asia are eliminated through the long term off-take and supply agreement. The culture of merchant facilities (i.e. spot purchase) has not been adopted at large in PPP power projects in this region. Moreover, it is also experienced that credit rating agencies have downgraded merchant power business in Asia due to power price volatility. This observation is reinforced and reflected in the outcome of the questionnaire survey by the respondents.

It is also necessary to identify the legal factors that commonly lead to successful setup of SPV for PPP projects in a country, and to incorporate these factors as criteria for predicting success in future PPP projects of a similar nature. Legal and institutional framework must be in place before setting up SPV for PPP projects. Historically, the lack of legislative and regulatory frameworks has hampered project financing in developing countries in Asia. In view of this, governments must decide by which active steps private sector investment and development can be achieved and identify the negative impact of existing institutional environment, political commitment and legal status. It is reflected in the analysis that, $41.9 \%$ and $45.2 \%$ of respondents gave highest weightage 
(i.e. $4=$ very important and $5=$ extremely important) to this factor. Lack of legal status for project financing and absence of government templates for PPP can lead to lengthy negotiation which in turn would hinder investors from setting up SPV for a project. Hence, the declared policy of the government with stable track record would help to mitigate political, legal and regulatory risks which could eventually ease the structuring of SPV for PPP projects.

\section{Conclusions}

Given the necessity for establishing SPV for PPP power projects in Asia, the purpose of this paper was to identify critical financial and legal factors through questionnaire survey and to take a critical look at these factors that have been identified to address the challenges/obstacles in implementing PPP power projects in Asia. The research findings show that, the factors of contractual foundation, enabling environmental, guarantee and credit enhancement (as shown in Table 3) need careful consideration (particularly the factors of higher ranking) in crafting SPV for PPP power projects in Asia so that the project can sustain against readily foreseeable policy and regulatory risks, market risk, counterparty risks and finally investment risk. For example, the private parties can evaluate government commitments and creditworthiness through 'establish an escrow agreement between SPV and off-taker to capture revenues from off-taker customer to support off-taker's payment obligation' factor. They should also be able to recognize the policy related factors may not be that significant on specific project by evaluating 'long track record of country's legal stability', 'well developed legal system and significant precedence', 'legal statutes for project finance', and 'law and bidding award' factors. Thus, the findings reported here will help the public sector in implementing PPP power policy development and private sector sponsors in managing the projects. However, in tailoring these criteria for a specific power project, adjustment should be made to reflect each party's role and capability in the project, because each factor has different weightage in SPV's setup which may affect the success of the PPP power projects.

\section{Limitation of the study and future research}

All the respondents were from Asia. It is found that the respondents had greater propensity to choose middle response categories due to Asian cultures and Confucian teachings regarding "the middle way" ( $\mathrm{Si}$, Cullen 1998). The influence of Confucianism and "the middle way" on Asian cultures (Hofstede 1991; Hofstede, Bond 1988) might have biased the respondents to select the central point that were found in survey instruments. To overcome such tendency, future research can be done on this study by avoiding explicit midpoint in odd number response categories.

\section{Acknowledgments}

The authors are grateful to all respondents of the survey for their valuable opinions and contributions. 


\section{References}

Asian Development Bank. Loans and Political Risk Guarantee Bangladesh: Meghnaghat Power Project, March 2008.

Blackwell, M. 2000. The PFI/PPP and Property. Chandos, Oxford, England.

Bult-Spiering, M.; Dewulf, G. 2006. Strategic Issues in Public-Private Partnerships: An International Perspective. Blackwell Publishing, Oxford, England.

Chowdhury, A. N.; Charoenngam, C. 2008. Special purpose vehicles for infrastructure project financing through Public Private Partnerships, in Davis, H. A. (Ed.). Financing Infrastructure Projects: Trends and Techniques, Euromoney Publication Plc. London, United Kingdom, 267-278.

Chowdhury, A. N.; Charoenngam, C. 2009. Factors influencing finance on IPP projects in Asia: a legal framework to reach the goal, International Journal of Project Management 27(1): 51-58. http://dx.doi.org/10.1016/j.ijproman.2008.01.011

Chowdhury, A. N.; Chen, P.-H. 2010. Special Purpose Vehicle (SPV) of Public Private Partnership Projects in Asia and Mediterranean Middle East: trends and techniques, International Journal of Institutions and Economies 2(1): 64-88.

Cooper, D. R.; Schindler, P. S. 2003. Business Research Methods. Singapore: McGraw-Hill.

Dailami, M.; Leipziger, D. 1997. Infrastructure project finance and capital flows, World Bank Policy Research Working Paper No. 1861. The World Bank, Washington D.C.

Davis, H. A. 2003. Project Finance: Practical Case Studies. Vol. 1. Euromoney Books. London, United Kingdom.

Delmon, J. 2009. Private Sector Investment in Infrastructure: Project Finance, PPP Projects and Risk. Kluwer Law International, the Netherlands.

Devapriya, K. A.; Alfen, H. W. 2003. Role of institutional arrangements in financing project companies in Asia, Working Paper. Knowledge Centre at Weimar, Banhaus- Universitat Weimar, Germany.

Dias, Jr. A.; Ioannou, P. G. 1995. Debt capacity and optimal capital structure for privately financed infrastructure projects, Journal of Construction Engineering and Management 121(4): 404-414. http://dx.doi.org/10.1061/(ASCE)0733-9364(1995)121:4(404)

Finnerty, J. D. 1996. Project Financing: Asset-Based Financial Engineering. New York: John Wiley \& Sons.

Fishbein, G.; Babbar, S. 1996. Private financing of toll roads, RMC Discussion Paper Series 117. The World Bank, Washington D.C.

Gatti, S. 2008. Project Finance in Theory and Practice: Designing, Structuring and Financing Private and Public Projects. Elsevier, London, England.

Hardcastle, C.; Boothroyd, K. 2003. Risks overview in public-private partnership, in Akintola Akintoye; Matthias Beck; Cliff Hardcastle (Eds.). Public-Private Partnerships: Managing Risks and Opportunities. United Kingdom: Blackwell Publishing, 31-57.

Hoffman, S. L. 2008. The Law and Business of International Project Finance. Cambridge: University Press, USA.

Hofstede, G. 1991. Cultures and Organizations: Software of the Mind. New York: McGraw-Hill.

Hofstede, G.; Bond, M. H. 1988. The Confucius connection: from cultural roots to economic growth, Organizational Dynamics 16(4): 5-21. http://dx.doi.org/10.1016/0090-2616(88)90009-5 International Project Finance Association, IPFA Supra Note 4 [cited 5 May 2008]. Available from Internet: http://www.dundee.ac.uk/cepmlp/car/assets/images/Bayo.pdf

Irwin, T.; Klein, M.; Perry, G. E.; Thobani, M. 1997. Dealing with public risk in private infra- 
structure: an overview, in Irwin, T. (Ed.). World Bank Latin American and Caribbean Studies Viewpoints: Dealing with Public Risk in Private Infrastructure. Washington, 1-19.

Jefferies, M.; Gameson, R.; Rowlinson, S. 2002. Critical success factors of the BOOT procurement system: reflection from the stadium Australia case study, Engineering, Construction and Architectural Management 9(4): 352-361.

Khan, M. F. K.; Parra, R. J. 2003. Financing Large Projects: Using Project Finance Techniques and Practices. Singapore: Pearson Prentice Hall.

Klein, M. 1997. Managing guarantee programs in support of infrastructure investment, World Bank Policy Research Working Paper No. 1812. The World Bank, Washington D.C.

Kumaraswamy, M. M.; Zhang, X. Q. 2001. Governmental role in BOT-led infrastructure development, International Journal of Project Management 19: 195-205.

http://dx.doi.org/10.1016/S0263-7863(99)00069-1

Kumaraswamy, M. M.; Zhang, X. Q. 2003. Risk assessment and management in BOT-type public-private partnership projects in China- with speed reference to Hong Kong, in Akintola Akintoye; Matthias Beck; Cliff Hardcastle (Eds.). Public-Private Partnerships: Managing Risks and Opportunities. United Kingdom: Blackwell Publishing, 263-283.

Lang, L. H. P. 1998. Project Financing in Asia. Elsevier, Amsterdam, the Netherlands.

Merna, A.; Smith, M. J. 1996. Guide to the Preparation and Evaluation of Build Own Operate Transfer Project Tenders. Asia Law and Practice Ltd., Hong Kong.

Qiao, L.; Wang, S. Q.; Tiong, R. L. K.; Chan, T. S. 2001. Framework for critical success factors of BOT projects in China, The Journal of Project Finance 7(1): 53-61.

Saleque, K. A. 2009. Tata wants to restart investment talks with Bangladesh [online], [cited 18 Oct 2010]. Energy Bangla. Available from Internet: http://www.energybangla.com/index.php?m od $=$ article \&cat $=$ SomethingtoSay\&article $=2304$

Sapte, W. 1997. Project Finance: the Guide to Financing Build-operate-transfer Project. Euromoney Publication PLC, Hong Kong.

Si, S. X.; Cullen, J. B. 1998. Response categories and potential cultural bias: effects of an explicit middle point in cross-cultural survey, International Journal of Organizational Analysis 6(3): 218-230. http://dx.doi.org/10.1108/eb028885

Smith, N.; Zhang, H.; Zhu, Y. 2004. The Huaibei power plant and its implication for the Chinese BOT market, International Journal of Project Management 22(1): 407-413.

http://dx.doi.org/10.1016/j.ijproman.2003.09.001

Standard and Poor's. 2001. Project and Infrastructure Finance Review. New York: The McGrawHill Co. Inc.

Tan, W. 2007. Principle of Project and Infrastructure Finance. New York: Taylor and Francis.

Tiong, R. L. K.; Alum, J. 1997. Evaluation of proposals for BOT projects, International Journal of Project Management 15(2): 67-72. http://dx.doi.org/10.1016/S0263-7863(96)00003-8

Tiong, R.; Anderson, J. A. 2003. Public-private partnership risk assessment and management process: the Asian dimension, in Akintola Akintoye; Matthias Beck; Cliff Hardcastle (Eds.). Public-Private Partnerships: Managing Risks and Opportunities. United Kingdom: Blackwell Publishing, 225-243.

Walsh, A. 2003. A legal perspective on risk management in public-private partnership, in Akintola Akintoye; Matthias Beck; Cliff Hardcastle (Eds.). Public-Private Partnerships: Managing Risks and Opportunities. United Kingdom: Blackwell Publishing, 153-181.

Wang, S. Q.; Tiong, L. K. 2000. Case study of government initiatives for PRC's BOT power plant project, International Journal of Project Management 18(1): 69-78.

http://dx.doi.org/10.1016/S0263-7863(98)00072-6 
Ye, S.; Tiong, R. K. L. 2000. Government support and risk-return trade-off in China's BOT power projects, Engineering Construction and Architectural Management 7: 412-422.

http://dx.doi.org/10.1046/j.1365-232x.2000.00175.x

Yescombe, E. R. 2002. Principles of Project Finance. United Kingdom: Academic Press.

Zhang, W. R.; Wang, S. Q.; Tiong, R. L. K.; Ting, S. K.; Ashley, D. 1998. Risk management of Shanghai's privately financed Yan'an Donglu tunnels, Engineering, Construction and Architectural Management 5(4): 399-409. http://dx.doi.org/10.1108/eb021092

Zhang, X. 2004. Concessionaire selection: methods and criteria, Journal of Construction Engineering and Management 130(2): 235-244.

http://dx.doi.org/10.1061/(ASCE)0733-9364(2004)130:2(235)

Abu Naser CHOWDHURY is currently a Ph.D. candidate of Nanyang Technological University, Singapore. Prior to that, he was a Research Associate of Asian Institute of Technology, Thailand. He has an MEng. from Asian Institute of Technology, Thailand and a B.Sc. from Bangladesh University of Engineering and Technology, Bangladesh. His research interest is on Public-Private Partnership (PPP). He has published several papers on PPP in various international journals such as Construction, Management and Economics, International Journal of Project Management, International Journal of Institutions and Economies and a book chapter in EuroMoney Publication.

Po-Han CHEN is currently an Associate Professor in the Department of Civil Engineering, National Taiwan University. Prior to that, he was on the faculty of School of Civil and Environmental Engineering, Nanyang Technological University, Singapore for eight years. He got his Ph.D. from Purdue University, U.S.A. and his research interests include Public Private Partnership (PPP), IT applications in construction, sustainable environment, and image processing.

Robert Lee Kong TIONG is an Associate Professor in the School of Civil \& Structural Engineering, Nanyang Technological University (NTU), Singapore. He has achieved B.Eng. (First Class Hons) degree in Civil Engineering from the University of Glasgow, Scotland, UK, and M.Eng. degree in Construction Management from the University of California, Berkeley, USA and Ph.D. degree in Civil Engineering from NTU, Singapore. He is extensively involved in teaching, research and consultancy on project financing, construction engineering and management especially on BOT projects. He has published about 30 journal papers, 20 conference papers, 2 books/monographs and some other papers. Besides, he is a member of American Society of Civil Engineers (ASCE) and a Professional Engineer (PE) of Singapore. 